\title{
Alternative surgical strategies in patients with sporadic medullary thyroid carcinoma: Long-term follow-up
}

\author{
SHI HONG MA ${ }^{1}$, QIN JIANG LIU ${ }^{2}$, YOU CHENG ZHANG ${ }^{1}$ and RONG YANG ${ }^{2}$ \\ ${ }^{1}$ Department of General Surgery, Lanzhou University Second Hospital; ${ }^{2}$ Department of Head-Neck Surgery \\ and Oncology, Gansu Province Tumor Hospital, Lanzhou, Gansu 730050, P.R. China
}

Received January 19, 2011; Accepted June 20, 2011

DOI: $10.3892 / \mathrm{ol} .2011 .344$

\begin{abstract}
The extent of surgical resection in patients with sporadic medullary thyroid carcinoma (SMTC) remains controversial. The aim of the present study was to discuss the prognosis of sporadic medullary thyroid carcinoma with different surgical treatments. Of 73 patients with SMTC (mean age of 43.78 years at diagnosis), 70 patients were followed up for 12.0-169.0 months (median 90.0). Having given their informed consent, 12 patients underwent total thyroidectomy with bilateral central neck dissection (group A), 40 underwent subtotal thyroidectomy preserving contralateral thyroid tissue on the entrance point of the recurrent laryngeal nerve into the larynx with ipsilateral modified radical neck dissection (group B), and 18 patients underwent subtotal thyroidectomy preserving contralateral thyroid tissue on the entrance point of the recurrent laryngeal nerve into the larynx with bilateral modified radical neck dissection (group C). The diagnosis was confirmed by a pathology examination. The incidences of hypoparathyroidism and recurrent laryngeal nerve injury, the cancer recurrence rates and survival time were investigated post-operatively. Significant differences were found between groups $\mathrm{A}, \mathrm{B}$ and $\mathrm{C}$ in the incidence of hypoparathyroidism $\left(\chi^{2}=40.9, P<0.01\right)$, as well as that of recurrent laryngeal nerve injury $\left(\chi^{2}=32.9, \mathrm{P}<0.01\right)$. The cancer recurrence rates in groups A, B and C were $75.0 \%(9 / 12), 2.5 \%(1 / 40)$ and $44.4 \%(8 / 18)$ respectively, $\left(\chi^{2}=31.1, \mathrm{P}<0.01\right)$ and the cure rates were $25,97.5$ and $55.6 \%$ respectively $\left(\chi^{2}=31.1, \mathrm{P}<0.01\right)$. The mean survival times in groups A, B and C were 77.8, 106.1 and 111.0 months respectively, but significant difference was noted $\left(\chi^{2}=3.2\right.$, $\mathrm{P}>0.05)$. In conclusion, compared to total thyroidectomy with bilateral central neck dissection, subtotal thyroidectomy with ipsilateral/bilateral modified radical neck dissection showed a lower incidence of hypoparathyroidism, recurrent laryn-
\end{abstract}

Correspondence to: Professor You Cheng Zhang, Department of General Surgery, Lanzhou University Second Hospital, Cuiyingmen 82, Chengguan, Lanzhou, Gansu 730030, P.R. China

E-mail: zhangychmd@yahoo.com.cn

Key words: medullary thyroid carcinoma, prognosis, thyroid surgery, thyroidectomy, follow-up geal nerve injury and lower rates of recurrence, along with a similar cumulative survival.

\section{Introduction}

Medullary thyroid carcinoma (MTC) arises from calcitonins (CT) produced by the parafollicular or C-cells. MTC accounts for $5-10 \%$ of all thyroid carcinoma, but is responsible for up to $13.4 \%$ of all deaths related to this disease (1-3). Approximately $75 \%$ of MTC cases are sporadic and $25 \%$ are hereditary. Sporadic medullary thyroid carcinoma (SMTC) usually presents as a unifocal clone population of tumor cells and clinically presents as a palpable thyroid tumor, with palpable neck lymph nodes in $30-60 \%$ of cases $(4,5)$. MTC readily invades the intraglandular lymphatics and regional lymph nodes. Distant metastases to the lung, bones and liver occur early during the course of the disease. Surgical treatment is the only curative treatment for SMTC with an acceptable morbidity, and all neoplastic foci present in the neck should be removed (7-9). In contrast to differentiated carcinoma arising from thyroid follicular cells, radioactive iodine and thyroid stimulating hormone suppression therapies are not effective for SMTC. Meticulous lymph node dissection was reported to result in a high biochemical cure rate $(10,11)$. Although total thyroidectomy with central neck dissection is recommended for SMTC patients, the indication for lateral neck dissection and total thyroidectomy remains controversial. Patients with known or highly suspected MTC with no evidence of advanced local invasion by the primary tumor, no evidence of cervical lymph node metastases on physical examination and cervical ultrasonography, and no evidence of distant metastases, should undergo total thyroidectomy and prophylactic central compartment (level VI) neck dissection $(12,13)$. Some surgeons advocate a bilateral neck dissection for both hereditary and sporadic MTC with unilateral palpable tumors $(10,14)$.

Previously, many general surgeons simply performed total thyroidectomy. However, since SMTC, which has usually already spread to the local lymph nodes at the time of presentation, has become more widely understood, a number of endocrine surgeons now advocate a total thyroidectomy and central lymph node dissection as the minimum initial operation. Some centers routinely add a modified radical neck dissection when the primary tumor is palpable or the lymph nodes are involved. Furthermore, the incidence of 
complications related to total thyroidectomy, including hypoparathyroidism and recurrent laryngeal nerve injury has yet to be evaluated. Thus, there is considerable confusion regarding standard surgical treatment of SMTC.

In the present retrospective study, we assessed 75 patients with SMTC who underwent the different surgical treatments including total thyroidectomy with bilateral central neck dissection (group A), subtotal thyroidectomy with ipsilateral modified radical neck dissection (group B) or subtotal thyroidectomy with bilateral modified radical neck dissection (group C) to identify the recurrence rate, cure rate, metastatic involvement of neck lymph nodes and incidences of surgically derived hypoparathyroidism and recurrent laryngeal nerve injury, and to evaluate the survival time between the different surgical treatment groups.

\section{Patients and methods}

Patients. Records of the 75 patients, 25 male and 45 female (mean 43.78 years) patients, with SMTC treated and/or followed at Gansu Province Tumor Hospital, China and Lanzhou University Second Hospital between January 1990 and December 2008 were reviewed. Of the 75 patients, 3 patients were excluded: 1 patient had metastasis to the bone and reported bone pain at presentation, and the remaining 2 presented metastasis to the lung and reported dyspnea at presentation. Based on the extent of surgical procedure, 70 patients were divided into groups A (12 patients, total thyroidectomy with bilateral central neck dissection), B (40 patients, subtotal thyroidectomy preserving contralateral thyroid tissue on the entrance point of the recurrent laryngeal nerve into the larynx with ipsilateral modified radical neck dissection) and C (18 patients, subtotal thyroidectomy preserving contralateral thyroid tissue on the entrance point of the recurrent laryngeal nerve into the larynx with bilateral modified radical neck dissection). SMTC was defined by the absence of rearranged during transfection (RET) proto-oncogene mutation, or (prior to the introduction of RET analysis) by a negative familial history of MTC and by a negative familial screening with a pentagastrin stimulation test.

Surgical treatment. The surgical procedure was performed with a curative intent for all SMTC patients. The pre-operative diagnosis of SMTC was performed in 4 patients for elevated pre-operative basal calcitonin levels and in 2 patients for elevated pentagastrin-stimulated calcitonin levels. The palpable bilateral neck lymph nodes were not present prior to the operation in any of the patients confirmed by the intra-operative frozen section analysis on thyroid tumor tissue and central neck lymph nodes and post-operative histopathology. In the patients of group A, the metastatic involvement of central neck lymph nodes was not revealed by the intra-operative frozen section analysis. In the patients of group B, the positive metastatic involvement of central lymph nodes was revealed by the intraoperative frozen section analysis. In the patients of group $\mathrm{C}$, metastatic involvement of ipsilateral lymph nodes was revealed by the intra-operative frozen section analysis. The central neck dissection constituted the removal of all soft tissue containing lymph nodes from the hyoid bone superiorly, to the innominate vessels inferiorly, and the jugular veins laterally (level
VI) (12). In $10 \%$ of patients with a macroscopic central node involvement, the upper mediastinal dissection was performed by cervical incision. In 2 patients, apparent surgical invasion of the trachea was treated by shaving the tumor from the trachea. The resection of an invaded recurrent laryngeal nerve was performed in 5 patients.

The subtotal thyroidectomy mainly preserved the thyroid tissue on the entrance point of the recurrent laryngeal nerve into the larynx and preserved the vessels supplying the parathyroid as far as possible.

The ipsilateral/bilateral modified radical neck dissection including the removal of the nodes around the spinal accessory nerve was performed (level II-V) (12). The lateral neck dissection routinely preserved the internal jugular vein, sternocleidomastoid muscle and spinal accessory nerve.

Parathyroid glands were identified with intra-operative methylene blue staining. Where a parathyroid gland was removed and was confirmed to be parathyroid tissue by frozen section analysis, the parathyroid gland was then sliced into 3 -mm sections and auto-transplanted into individual pockets in the sternocleidomastoid muscle, or in a muscle of the non-dominant forearm. Auto-transplanted procedures were performed in accordance with the ethical guidelines of our institution.

Surgical complications. Laryngeal examination was regularly performed post-operatively whether a laryngeal nerve palsy was present or not. Serum calcium, phosphorus and parathyroid hormone were periodically measured until normalization occurred. Where laryngeal nerve palsy and hypoparathyroidism lasted for more than 1 year after surgery, diagnoses of operation-related complications were finally established.

Pathological diagnosis. Patients were diagnosed by intra-operative frozen section analysis on thyroid tumor tissue and central neck lymph nodes. The diagnosis of MTC was confirmed by a positive immunostaining for calcitonin and carcino-embryonic antigen in all patients. The characteristics of the tumor, including the location (upper, central-upper, or lower part of the thyroid lobe), the size, uni- or multifocality, and uni- or bilaterality were assessed by an experienced pathologist. Sections (2-mm) of each lymph node without apparent metastases were routinely performed. In case of macroscopic metastases, fewer sections were performed. In case of doubtful diagnosis of micrometastases, calcitonin and carcino-embryonic antigen immunostaining was performed on the lymph node sections. No attempt was made to classify central lymph nodes as right or left since this compartment was removed in monoblock. The latero-cervical lymph nodes were classified as right or left. In case of the bilateral thyroid involved, the contralateral lymph nodes were defined as contralateral to the largest tumor.

Criterion of therapeutic outcome. At the point of 3 months after surgery, assessment of the basal calcitonin was determined. Where the basal calcitonin was undetectable, a pentagastrin stimulation test was performed (slow intravenous injection of $0.5 \mu \mathrm{g} / \mathrm{kg}$ pentagastrin-peptavlon, and peak calcitonin measured at 2 and $5 \mathrm{~min})(15)$.

Statistical analysis. Data are described as the mean \pm SD. ANOVA was used to compare the number of involved lymph 
Table I. The characteristics of 70 patients with SMTC.

\begin{tabular}{lccc}
\hline Group & No. $(\%)$ & Age $(\mathrm{yrs})$ & Gender (male/female) \\
\hline A & $12(17.2 \%)$ & $46.8 \pm 14.4$ & $7 / 5$ \\
B & $40(57.1 \%)$ & $44.3 \pm 13.5$ & $10 / 30$ \\
C & $18(25.7 \%)$ & $39.4 \pm 13.0$ & $10 / 8$ \\
$\chi^{2}$ & & & 8.12 \\
P-value & & $1.0>0.05$ & $0.018<0.05$ \\
\hline
\end{tabular}

SMTC, sporadic medullary thyroid carcinoma. Group A: total thyroidectomy with central neck dissection. Group B: subtotal thyroidectomy with ipsilateral modified radical neck dissection; Group C: subtotal thyroidectomy with bilateral modified radical neck dissection.

nodes, tumor size and survival time among the different groups. A $\chi^{2}$ test was used to evaluate the incidence of surgical complications, recurrence rate, cure rate and TNM stage among the different groups. The Kaplan-Meier method and the log-rank test were used to determine the cancer-specific cumulative survival. $\mathrm{P}<0.05$ was considered to be statistically significant.

\section{Results}

Follow-up was available for 70 patients administered with three different surgical treatment types. Among 70 patients, 50 cases $(71.4 \%)$ had undergone different initial thyroid surgeries, including lobe thyroidectomy, lobe subtotal thyroidectomy and lobe partial thyroid resection in the community hospital. Thyroid nodules were detected by palpation in 60 cases and by ultrasonography in 10 cases. The mean age at diagnosis was 43.78 years without a significant difference between the 3 groups. There were 25 males and 45 females and the overall male-to-female ratio was $1.0: 1.8$ with a significant difference between the 3 groups. Patients with SMTC were diagnosed by intra-operative frozen section analysis and post-operative pathological examination of thyroid tumor tissue and central neck lymph nodes. The clinical characteristics of the 70 patients are reported in Table I.

Cancer recurrence rates. The tumor sizes were $8.3 \pm 5.3$, $3.3 \pm 1.5$ and $4.8 \pm 0.4 \mathrm{~cm}$, in groups $\mathrm{A}, \mathrm{B}$ and $\mathrm{C}$ respectively. A significant difference was observed between groups $\mathrm{A}$ and $\mathrm{B}$ $(\mathrm{P}=0.03)$, whereas no difference was found between groups $\mathrm{A}$ and $\mathrm{C}(\mathrm{P}>0.05)$, or groups $\mathrm{B}$ and $\mathrm{C}(\mathrm{P}>0.05)$. A significant difference was not observed in the number of neck lymph node metastases (A, 0.86 $\pm 1.46 ; \mathrm{B}, 2.55 \pm 4.38$; and $\mathrm{C}, 7.5 \pm 5.3$ ) and the survival (A, 70 $\pm 49.8 ; \mathrm{B}, 92 \pm 42.7$ and $\mathrm{C}, 90.7 \pm 26.9)$ among the 3 groups $(\mathrm{P}>0.05)$. The tumor size, number of neck lymph node metastases and survival time of 70 patients are reported in Table II.

No differences were found in the clinical and pathological stage according to the TNM classification in the 3 groups ( $P>0.05)$. Notably, 2 patients exhibited concomitant occult papillary thyroid carcinoma and 1 patient exhibited concomitant follicular thyroid carcinoma. Symptoms such as flushing, diarrhea and bone pain were concomitantly presented by 5 patients.

\section{Survival functions}

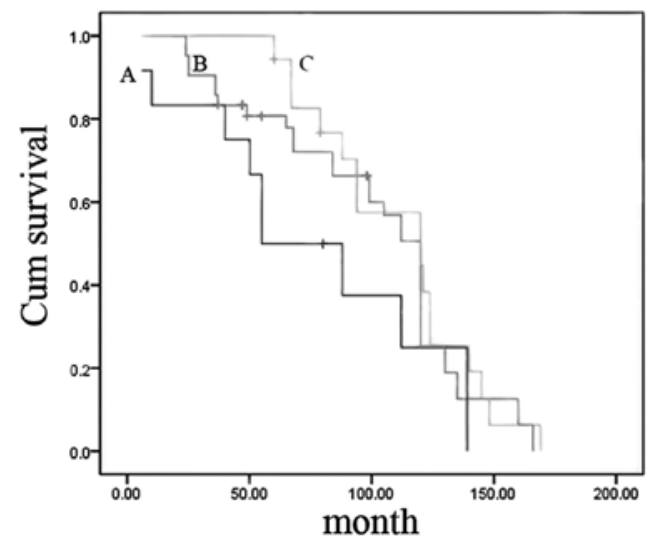

Figure 1. Cancer-specific cumulative survival by Kaplan-Meier analysis is shown for 70 patients with SMTC. The overall cause-specific cumulative survival observed in our study was 130 months (25\%), 112 months (50\%), and 67 months $(75 \%)$. The mean survival times were $77.8,106.1$ and 111.0 months in groups $\mathrm{A}, \mathrm{B}$ and $\mathrm{C}$, respectively. No significant difference was observed in survival time by Kaplan-Meier analysis.

Family history was not recorded for any of the MTC patients. During the follow-up (12.0-169.0 months), 1 patient presented with metastasis to the lung and 1 to bone $(2.8 \%)$. The overall incidence of recurrence was $25.7 \%$ (18/70), with recurrence site at the ipsilateral neck in 10 patients, contralateral neck lymph nodes in 4 patients and thyroid bed in 4 patients. Among 18 recurrent cases, 9 patients were distributed in group A, 1 patient in group $B$ and 8 patients in group $C$. The biochemical cure rate in groups $\mathrm{A}, \mathrm{B}$ and $\mathrm{C}$ was found to be $25,97.5$ and $55.6 \%$, respectively. The possibility of re-operation in group A was greater than that in the remaining 2 groups. A significant difference was observed between the 3 groups with regard to the recurrence $\left(\chi^{2}=31.1, \mathrm{P}<0.001\right)$ and biochemical cure rates $\left(\chi^{2}=31.1, \mathrm{P}<0.01\right)$. The cancer recurrence rates in groups A, B and C were 75.0, 2.4 and $44.4 \%$, respectively $\left(\chi^{2}=31.1\right.$, $\mathrm{P}<0.01)$. The biochemical cure rates were 25, 97.5 and $55.6 \%$, respectively $\left(\chi^{2}=31.1, \mathrm{P}<0.01\right)$. The recurrence and cure rates of 70 patients with SMTC are reported in Table III.

Surgical complications. Neck surgery was considered to be macroscopically complete in 70 patients. Postoperative complications included permanent hypoparathyroidism in 4 cases ( 3 cases after initial thyroidectomy performed at primary hospital) and transient or momentary hypoparathyroidism in 7 cases (these patients required maintenance therapy of oral calcium in the post-operative 6 months) 11/70 (15.7\%). Recurrent laryngeal nerve injury was observed in 8 patients $(11.1 \%)$ and of these, recurrent nerve resection was intentionally performed in 5 patients to achieve complete tumor resection. A significant difference was observed in the incidences of hypoparathyroidism $\left(\chi^{2}=40.9, \mathrm{P}<0.01\right)$ and recurrent laryngeal nerve injury $\left(\chi^{2}=32.9, \mathrm{P}<0.01\right)$ among the 3 surgical groups (Table IV). One patient presented with Claude Bernard-Horner syndrome and 1 patient presented with spinal nerve palsy.

Survival analysis. Therapeutic external beam radiation to the cervical region was administered to 8 patients, and 4 patients received chemotherapy regimens ( 2 patients received systemic 
Table II. Tumor size, number of neck lymph node (NLN) metastases and survival of 70 patients.

\begin{tabular}{|c|c|c|c|c|c|c|}
\hline & \multicolumn{4}{|c|}{ Groups } & \multirow[b]{2}{*}{$\mathrm{C}$} & \multirow[b]{2}{*}{ P-value } \\
\hline & A & P-value & B & P-value & & \\
\hline No. $(\%)$ & $12(17.2 \%)$ & & $40(57.1)$ & & $18(25.7)$ & \\
\hline Tumor size $(\mathrm{cm})$ & $8.3 \pm 5.3$ & $0.03^{\mathrm{a}}$ & $3.3 \pm 1.5$ & $0.88^{\mathrm{b}}$ & $4.8 \pm 0.4$ & $1.0^{\mathrm{c}}$ \\
\hline No. of NLN metastases & $0.86 \pm 1.46$ & $0.8^{\mathrm{a}}$ & $2.55 \pm 4.38$ & $0.37^{\mathrm{b}}$ & $7.5 \pm 5.3$ & $0.07^{\mathrm{b}}$ \\
\hline Survival period (month) & $70 \pm 49.8$ & $1.0^{\mathrm{a}}$ & $92 \pm 42.7$ & $1.0^{\mathrm{b}}$ & $90.7 \pm 26.9$ & $1.0^{\mathrm{b}}$ \\
\hline
\end{tabular}

Table III. Recurrence and cure rate of 70 patients with SMTC.

\begin{tabular}{lcccccc}
\hline & \multicolumn{7}{c}{ Groups } & & \\
\cline { 2 - 5 } & A & B & C & Total & $\chi^{2}$ & P-value \\
\hline No. (\%) & $12(17.2)$ & $40(57.1)$ & $18(25.7)$ & 72 & & \\
Recurrence (\%) & $9(75)$ & $1(2.5)$ & $8(44.4)$ & $18(25.7)$ & 31.09 & $<0.001$ \\
Re-operation (\%) & $9(75)$ & $1(2.5)$ & $8(44.4)$ & $18(25.7)$ & 31.09 & $<0.001$ \\
Cure (\%) & $3(25)$ & $39(97.5)$ & $10(55.6)$ & $52(74.3)$ & 31.09 & $<0.001$ \\
\hline
\end{tabular}

SMTC, sporadic medullary thyroid carcinoma. Group A: total thyroidectomy with central neck dissection; Group B: subtotal thyroidectomy with ipsilateral modified radical neck dissection; Group C: subtotal thyroidectomy with bilateral modified radical neck dissection.

chemotherapy and 1 patient had chemoembolization for liver metastases post-operation). The overall cause-specific cumulative survival observed in our study was 130 months $(25 \%)$, 112 months (50\%), and 67 months (75\%). The mean survival times in groups A, B and C were 77.8, 106.1 and 111.0 months, respectively. No significant difference was observed in survival time by Kaplan-Meier analysis (Fig. 1) and among 3 surgical treatment groups $\left(\chi^{2}=3.2, \mathrm{P}>0.05\right)$ (Table II).

\section{Discussion}

Medullary thyroid carcinoma is a rare calcitonin-producing neuroendocrine tumor originating from the parafollicullar C-cells of the thyroid gland. This typically aggressive tumor presents with metastasis to local and regional lymph nodes and distant organs, particularly the lungs, liver and bone. MTC does not generally respond to external beam radiation, although chemotherapy agents and external beam radiation therapy for unresectable or metastatic MTC have produced various outcomes without a demonstrable improvement in long-term survival. At present, the only possible curative treatment against MTC is complete surgical resection when the disease is confined to the neck. However, this option is limited since a complete resection of MTC may be difficult. Consequently, surgical treatment remains the curative modality primarily employed for MTC (15). MTC occurs in sporadic and hereditary forms; the present study excluded hereditary medullary thyroid carcinoma (HMTC) for characteristics of HMTC with multicentric and heterogeneous clinical behavior. The present study included of 70 consecutive STMC patients receiving different surgical treatments including total thyroidectomy with central neck dissection (group A), subtotal thyroidectomy with ipsilateral modified radical neck dissection (group B) and subtotal thyroidectomy with bilateral modified radical neck dissection (group C). The mean age at diagnosis was 44.8 years, which was older than in other studies $(16,17)$. This difference may be due to the fact that most patients were diagnosed at a late stage. The overall male-to-female ratio was 1.0:1.8, indicating that the incidence of SMTC was higher in females than in males. Taken together, these findings contradict those of Jimenez et al, who observed that this tumor most commonly manifests equally in women and men in the fourth decade of life (18).

In accordance with previous data, a strong relationship was noted between lymph node involvement and tumor size in the 2 groups of MTC $(19,20)$. Patients presenting with a palpable nodule $(>1 \mathrm{~cm})$ were at a high risk of having ipsilateral or contralateral lymph node metastases. In our series, the tumor size of all of patients was $>1 \mathrm{~cm}$ and there were significant differences in tumor size between groups A and B, whereas the differences in lymph node metastasis between different surgical treatment groups were not significant. This observation indicates that the same surgical protocol should also be applied to patients with different tumor size where 
Table IV. The incidence of surgical complication of 70 patients with SMTC.

\begin{tabular}{|c|c|c|c|c|c|c|}
\hline & \multicolumn{4}{|c|}{ Groups } & \multirow[b]{2}{*}{$\chi^{2}$} & \multirow[b]{2}{*}{ P-value } \\
\hline & A & $\mathrm{B}$ & $\mathrm{C}$ & Total & & \\
\hline No. $(\%)$ & $12(17.2)$ & $40(57.1)$ & $18(25.7)$ & 72 & & \\
\hline Hypoparathyroidism (\%) & $9(75)$ & 0 & $2(11)$ & $11(15.3)$ & 40.882 & $<0.001$ \\
\hline $\begin{array}{l}\text { Recurrent laryngeal } \\
\text { nerve injury }(\%)\end{array}$ & $7(58.3)$ & 0 & $1(1.4)$ & $8(11.1)$ & 32.906 & $<0.001$ \\
\hline
\end{tabular}

the surgical procedure cannot be determined according to tumor size. Furthermore, the result clearly demonstrated that neck lymph node metastasis does not behave according to the conventional route (from level VI-VII to level II-V), but follows a skipping route (from tumor to level II-V).

The intra-operative palpation of nodes was not an accurate predictor of the presence or absence of metastases $(13,21)$. From data on lymphatic dissemination of MTC $(22,23)$, the ipsilateral cervical lymph node compartments constitute the border between local and systemic disease. Contralateral lymphatic drainage of tumor cells is indicative of systemic disease, as evidenced by the frequent failure of the calcitonin level to normalize despite extensive lymph node dissection. The appropriate surgical procedure for a patient with localized SMTC is a total thyroidectomy with central (levels VI and VII) and bilateral (levels II to V) lymph node dissections. Although a surgical cure is difficult to establish, up to $25 \%$ of selected patients can be cured by this approach (22-24). It was difficult to determine an appropriate surgical procedure for a patient with SMTC.

Findings of our study showed that of the 18 recurrences (25\%), 10 cases occurred at the ipsilateral neck lymph node, 4 cases occurred in the thyroid bed and 4 cases occurred at the contralateral neck lymph nodes. As a result all of the patients involved underwent reoperation. The recurrence rates were 75 , 2.5 and $44.4 \%$ for groups A, B and C, respectively, and the respective biochemical cure rates were $25,97.5$ and $55.6 \%$. A significant difference was observed between the 3 groups in the post-operative recurrence rate. Recurrence was more frequent among patients with definite metastases on central neck lymph nodes (levels VI and VII) who had not undergone ipsilateral/ bilateral neck dissection, but only central neck dissection. Our results showed that central neck dissection was insufficient for SMTC patients when intraoperative frozen section revealed metastatic involvement of the central neck lymph node.

Conversely, it was found that that total thyroidectomy with central neck dissection did not decrease recurrence even if the primary tumor was less than $2.0 \mathrm{~cm}$ in diameter. Kebebew et al observed that the extent of thyroidectomy was not an independent predictor of mortality (24). Incomplete and inadequate neck lymph node dissection may be the main reason for recurrence. By contrast, the cure rate of group B was significantly higher than that of the 2 groups, which showed inadequate neck dissection (group A) and advanced stage (group C) leading to recurrence and metastases. Macro- scopically complete neck surgery was required for those cases with bilateral neck lymph node metastasis. The invasively malignant biological behaviour resulted in patients requiring further surgery and adjunctive radiotherapy. Occasionally, palliative surgery was required, particularly where the tumor had invaded the tracheal, esophageal and/or carotid sheath. The extent of the central neck dissection includes all fibrofatty and lymphatic tissue from just above the innominate vein between the carotid sheath and trachea to the level of the thyroid cartilage, and a modified radical neck dissection preserving the internal jugular vein, the sternocleidomastoid muscle and the spinal accessory nerve, but removing all fibrofatty and lymphatic tissue from the carotid sheath to the trapezius and from the clavicle to just above the hyoid bone.

Radiotherapy is routinely used as an adjunctive, palliative treatment for extensive neck or mediastinal disease or for localized bony metastasis. Although radiotherapy appears to be effective in preventing and controlling complications associated with MTC activity in the neck and mediastinum, no evidence exists showing that such a therapy has a positive effect on survival (26). Chemotherapy based on dacarbazine has been associated with a significant reduction in tumor size in approximately $30 \%$ of patients treated with this agent in combination with others. However, neither a complete remission nor any effect on survival rates have been observed (27). In the present study, surgical complications resulted in hypoparathyroidism in $15.3 \%$ and recurrent laryngeal nerve injury in $11 \%$ of patients. Complication rates were higher than in other studies $(28,29)$. Complication rates of hypoparathyroidism and recurrent laryngeal nerve injury in group A were significantly higher than those in groups B and C, indicateing that total thyroidectomy with central neck dissection easily led to hypoparathyroidism and recurrent laryngeal nerve injury.

A number of reasons may explain this result. First, most cases had been operated by a non-standard initial thyroid surgery, which led to fibrous tissue with recurrent laryngeal nerve-wide adhesions and difficult anatomical separation. Confronted with such a situation, a surgeon may injure the supplying vascular of the parathyroid or dissect the parathyroid instead of the neck lymph node metastases. Additionally, intra-operative gross operating and separation may cause hypoparathyroidism and recurrent laryngeal nerve injury. Second, recurrent laryngeal nerve injury cannot be avoided especially in the case of re-operation or if the recurrent laryn- 
geal nerve has been invaded by a tumor, as in the 7 cases of the present study. Recurrent laryngeal nerve resection was intentionally performed in 5 patients to achieve complete tumor resection. In those cases where the thyroid tissue on the entrance point of the recurrent laryngeal nerve into the larynx had been invaded by a tumor it was necessary to select between the residual tumor or permanent recurrent laryngeal nerve palsy. Third, the variation in location, number and supplying vessel of the parathyroid may lead to post-operative hypoparathyroidism. Total thyroidectomy with central neck dissection only preserved the parathyroid, naked trachea and tracheo-esophageal groove. Conversely, subtotal thyroidectomy preserved maximum thyroid tissue on the entrance point of the recurrent laryngeal nerve into the larynx and the supplying vessel of the parathyroid; thus groups $\mathrm{B}$ and $\mathrm{C}$ showed a lower incidence of surgical complication. The mean survival times were 77.8, 106.1 and 111.0 months for groups A, B and C, respectively. This result was slightly poorer than the findings of other studies (30). No significant difference was found in the cumulative survival among the 3 groups, indicating that the 3 groups had a similar clinical effect, although group had a higher incidence of surgical complication and recurrence than groups B and C. In other words, the treatment administered to group A had not prolonged the survival time of SMTC patients through the adoption of total thyroidectomy with central neck dissection, but instead led to a higher incidence of recurrence and surgical complication, particularly that of hypoparathyroidism and recurrent laryngeal nerve injury.

In conclusion, our data provide a challenge to the standard approach to neck surgery on patients with SMTC, regardless of the size of the thyroid tumor and the incidence of lymph node metastases in the central and lateral neck compartments. Total thyroidectomy with central neck dissection presented a significantly higher surgical complication rate of hypoparathyroidism and recurrent laryngeal nerve injury and incidence of recurrence. Adoption of subtotal thyroidectomy preserving the thyroid tissue on the entrance point of the recurrent laryngeal nerve into the larynx with ipsilateral/bilateral modified radical neck dissection provided a more effective surgical strategy that showed a higher cure rate and lower complication rate.

\section{References}

1. Gilliland FD, Hunt WC, Morris DM and Key CR: Prognostic factors for thyroid carcinoma: a population-based study of 15 698 cases from the Surveillance, Epidemiology and End Results (SEER) program, 1973-1991. Cancer 79: 564-573, 1997.

2. Hundahl SA, Fleming ID, Fremgen AM and Menck HR: A national cancer database report on 53, 856 cases of thyroid carcinoma treated in the US, 1985-1995. Cancer 83: 2638-2648, 1998.

3. Marsh DJ, Learoyd DL and Robinson BG: Medullary thyroid carcinoma: recent advances and management update. Thyroid 5: 407-424, 1995.

4. Rougier P, Parmentier C, Laplanche A, et al: Medullary thyroid carcinoma: prognostic factors and treatment. Int J Radiat Oncol Biol Phys 9: 161-169, 1983.

5. Saad MF, Ordonez NG, Rashid RK, et al: Medullary carcinoma of the thyroid. A study of the clinical features and prognostic factors in 161 patients. Medicine (Baltimore) 63: 319-342, 1984.

6. Ellenhorn JD, Shah JP and Brennan MF: Impact of therapeutic regional lymph node dissection for medullary carcinoma of the thyroid gland. Surgery 114: 1078-1081, 1993.

7. Evans DB, Fleming JB, Lee JE, Cote G and Gagel RF: The surgical treatment of medullary thyroid carcinoma. Semin Surg Oncol 16: 50-63, 1999.
8. Fleming JB, Lee JE, Bouvet M, et al: Surgical strategy for the treatment of medullary thyroid carcinoma. Ann Surg 230: 697-707, 1999.

9. Moley JF and DeBenedetti MK: Patterns of nodal metastases in palpable medullary thyroid carcinoma: recommendations for extent of node dissection. Ann Surg 229: 880-887, 1999.

10. Dralle H, Damm I, Scheumann GF, Kotzerke J, Kupsch E, Geerlings $\mathrm{H}$ and Pichlmayr R: Compartment-oriented microdissection of regional lymph nodes in medullary thyroid carcinoma. Surg Today 24: 112-121, 1994.

11. Robbins KT: Pocket Guide to Neck Dissection Classification and TNM Staging of Head and Neck Cancer. American Academy of Otolaryngology Head and Neck Surgery Foundation Inc. Virginia, USA, pp9-20, 1991.

12. American Thyroid Association Guidelines Task Force, Kloos RT, Eng C, Evans DB, et al: Medullary thyroid cancer: management guidelines of the American Thyroid Association. Thyroid 19: 565-612, 2009.

13. Machens A, Hinze R, Thomusch O and Dralle H: Pattern of nodal metastasis for primary and reoperative thyroid cancer. World J Surg 26: 22-28, 2002.

14. Weber T, Shilling T and Buchler MW: Thyroid carcinoma. Curr Opin Oncol 18: 30-35, 2006.

15. Barbot N, Calmettes C, Schuffenecker I, et al: Pentagastrin stimulation test and early diagnosis of medullary thyroid carcinoma using an immunoradiometric assay of calcitonin: comparison with genetic screening in hereditary medullary thyroid carcinoma. J Clin Endocrinol Metab 78: 114-120, 1994.

16. Dottorini ME, Assi A, Sironi M, Sangalli G, Spreafico G and Colombo L: Multivariate analysis of patients with medullary thyroid carcinoma: prognostic significance and impact on treatment of clinical and pathologic variables. Cancer 77: 1556-1565, 1996.

17. Sherman SI, Brierley JD, Sperling M, et al: Prospective multicenter study of thyroid carcinoma treatment: initial analysis of staging and outcome. National Thyroid Cancer Treatment Cooperative Study Registry Group. Cancer 83: 1012-1021, 1998.

18. Jiménez C, Hu MI and Gagel RF: Management of medullary thyroid carcinoma. Endocrinol Metab Clin North Am 37: 481-496, 2008.

19. Beressi N, Campos JM, Beressi JP, et al: Sporadic medullary microcarcinoma of the thyroid: a retrospective analysis of eighty cases. Thyroid 8: 1039-1044, 1998.

20. Gimm O, Ukkat J and Dralle H: Determinative factors of biochemical cure after primary and reoperative surgery for sporadic medullary thyroid carcinoma. World J Surg 22: $562-567,1998$.

21. Quayle FJ and Moley JF: Medullary thyroid carcinoma: including MEN 2A and MEN 2B syndromes. J Surg Oncol 89: 122-129, 2005.

22. Machens A, Gimm O, Ukkat J, Hinze R, Schneyer U and Dralle H: Improved prediction of calcitonin normalization in medullary thyroid carcinoma patients by quantitative lymph node analysis. Cancer 88: 1909-1915, 2000.

23. Cohen Ms and Moley JF: Surgical treatment of medullary thyroid carcinoma. J Intern Med 253: 616-26, 2003.

24. Kebebew E, Ituarte PH, Siperstein AE, Duh QY and Clark OH: Medullary thyroid carcinoma. Clinical characteristics, treatment, prognostic factors, and a comparison of staging systems. Cancer 88: 1139-1148, 2000.

25. Kaltsas GA, Besser Gm and Grossman AB: The diagnosis and medical management of advanced neuroendocrine tumors. Endocr Rev 25: 458-511, 2004.

26. Monsieurs M, Brans B, Bacher K, Dierckx R and Thierens H: Patient dosimetry for 131I-MIBG therapy for neuroendocrine tumours based on 123I-MIBG scans. Eur J Nucl Med Mol Imaging 29: 1581-1587, 2002.

27. Moley JF, Dilley WG and DeBenedetti MK: Improved results of cervical reoperation for medullary thyroid carcinoma. Ann Surg 225: 734-740, 1997.

28. Shaha AR: Management of the neck in thyroid cancer. Otolaryngol Clin North Am 31: 823-831, 1998.

29. Machens A, Hofmann C, Hauptmann S and Dralle $\mathrm{H}$ : Locoregional recurrence and death from medullary thyroid carcinoma in a contemporaneous series: 5-year results. Eur J Endocrinol 157: 85-93, 2007.

30. Ito Y, Miyauchi A, Yabuta T, et al: Alternative surgical strategies and favorable outcome in patients with medullary thyroid carcinoma in Japan: experience of a single institution. World J Surg 33: 58-66, 2009. 